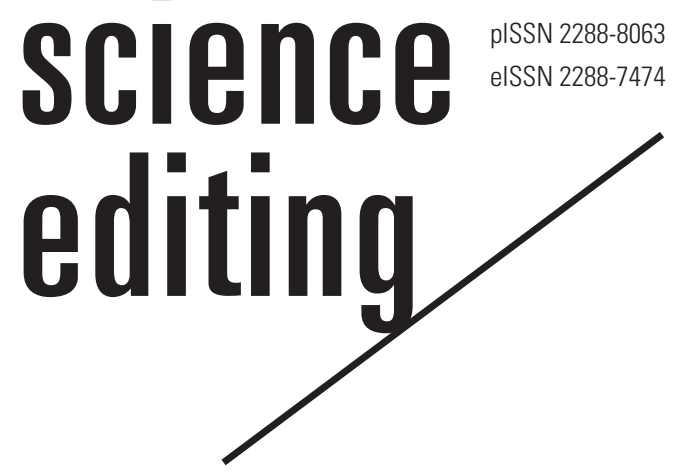

\title{
How to romanize Korean characters in international journals
}

\author{
Sun Huh \\ Department of Parasitology and Institute of Medical Education, College of Medicine, Hallym University, Chuncheon, Korea
}

\begin{abstract}
For editors and manuscript editors, the romanization of Korean characters is a topic that should be understood thoroughly, because Korean proper nouns have become more widely used worldwide due to phenomena such as Hallyu (the Korean wave). In this report, I describe the 2 major romanization systems used in Korea: the Korean government's romanization system and the McCune-Reischauer system. I also describe the transliteration guidelines presented in a variety of reference styles, such as the CSE (Council of Science Editors), ACS (American Chemical Society), AMA (American Medical Association), APA (American Psychological Association), IEEE (Institute of Electrical and Electronics Engineers) styles and the NLM (National Library of Medicine) style guide. I found that 2 journals have adopted the Korean government's romanization system, while 10 use the McCune-Reischauer system. Other journals do not specifically mention a romanization system. Editors should select a romanization system and use it consistently. When presenting a reference that includes romanized text, the journal's house style should be followed, based on international reference citation styles. Chinese characters in documents published in Korea should be romanized according to the Korean pronunciation.
\end{abstract}

Keywords

Editors; Korean language; Reference style; Republic of Korea; Romanization

Received: July 27, 2017

Accepted: August 1, 2017

Correspondence to Sun Huh shuh@hallym.ac.kr

ORCID

Sun Huh

http://orcid.org/0000-0002-8559-8640

\section{Introduction}

For Korean researchers, romanizing Korean characters is mandatory when submitting their research findings to international journals in languages that use Roman characters, such as English, German, French, and Spanish. Moreover, editors of international journals should provide guidelines for the romanization of non-Roman characters, such as Arabic, Greek, Russian, Chinese, Japanese, and Korean. In particular, scholarly journal editors in Korea should comprehend the romanization system of Korean characters because geographic features, cultural properties, man-made structures, administrative units, proper names such as personal names and those of companies, historical government posts, and historical publications should be de- 
scribed in Roman characters, not only in the main text but also in the references section. Therefore, in this article, I would like to present 2 romanization systems used for Korean characters and to discuss reference citation styles for publications. Although this information has already been published in a variety of style books and websites, this will be a helpful guideline for editors to study in a more intensive manner.

\section{Common Questions from Editor Colleagues}

I sometimes receive the following questions on the romanization of Korean:

1. There are 2 kinds of romanization used in Korea: one is the McCune-Reischauer system invented in 1939, and the other is the 2000 edition of Revised Romanization of Korean (the Korean government's Romanization). Which romanization standard should a science editor select?

2. If a document has in Koran only, should it be presented with only romanization or with both romanization and English translation in the references section?

3. If a document is in Chinese characters and was published in China or Japan, how should it be romanized?

4. If a document is in Chinese characters and was published in Korea, how should it be romanized?

I would like to answer these questions according to present standards and common sense.

\section{Two Ways to Romanize Korean Characters}

One method of romanization was promulgated as a regulation by the Korean government in 2000 [1]. The basic principle of this regulation is as follows: first, romanization is based on standard Korean pronunciation; second, symbols other than standard Roman letters are avoided to the greatest extent possible. This method does not precisely reflect the pronunciation of Korean characters, but does reflect reciprocal transliteration between Korean and English. Therefore, vowels are transcribed using a set of characters shared with English. Additionally, consonants are transcribed in a fixed form, except ᄀ, ᄃ, ㅂ, and ᄅ.

Another very commonly used method of romanization is the McCune-Reischauer system [2]. It is believed that at all international journals outside of Korea, the romanization of Korean characters follows this system. Furthermore, most social science and arts and humanities journals published in an English-language only format in Korea have also adopted the McCune-Reischauer system. However, exceptions include the Journal of Educational Evaluation for Health Professions and Korea Journal (ISSN 0023-3900). Those 2 journals follow the Korean government's guidelines. The Journal of Educational
Evaluation for Health Professions published the following editorial comment [3]:

For the romanization of Korean characters, all terms are spelled out according to the Korean Government Ministry of Culture and Tourism's Notice of Regulation 2000-8 (July 7, 2000) available from http://www.korean.go.kr/.

\section{Manuscript style regulations}

Please use the McCune-Reischauer system as the primary system to romanize Korean-language names and terms.

Other journals that have announced that they use the McCune-Reischauer system are listed in Table 1. It is difficult to find journals that have clearly indicated the method for romanizing Korean characters, except for the 10 journals presented in Table 1. As most journals have adopted the McCune-Reischauer system, the reason why there is no clear indication about romanization is believed to be that the McCune-Reischauer system is most generally accepted worldwide.

The most important differences between the Korean government's romanization system and the McCune-Reischauer system are as follows: first, the latter system introduced special symbols to indicate the pronunciation of Korean vowels more precisely. For example, there are no English vowels pronounced similarly to $\dashv$ and - . Therefore, they are transliterated as $\breve{o}$ and $\breve{u}$. The consonants $\exists$, $E$, and $\bar{\nabla}$ are transliterated as $k^{\prime}, t^{\prime}$, and $p^{\prime}$. However, the former system does not introduce any special symbols; therefore, -1 and - are transcribed as eo and eu. Second, the latter system more closely reflects real pronunciation. In particular, it differentiates voiceless sounds and voiced sounds straightforwardly. 가다 can be written as kada. However, in the former system it is written as gada. $\neg$ in 가 is a voiceless sound. Therefore, the latter system indicates the exact pronunciation; kada is better, because $k$ is a voiceless sound, while $g$ is a voiced sound. The same point applies to the distinct between $t$ (a voiceless sound) and $d$ (a voiced sound). The major problem in the McCune-Reischauer system is that the special symbols are difficult to use with an ordinary keyboard. The symbols $\breve{o}$ and $\breve{u}$ must be typed after searching for symbols in the extended Latin character group. Doing this is very difficult in realworld conditions, meaning that these 2 special symbols are often written simply as $o$ and $u$. Of course, some confusion will arise if $o$ is used for both $\dashv$ and $\perp$, because $o$ corresponds to the vowel $\perp$ in Korean. The value of the McCuneReischauer system is limited when special symbols are omitted. 
Table 1. Ten journal titles which announced the romanization system as McCune-Reischauer system

\begin{tabular}{|c|c|c|c|}
\hline Journal title & Publisher & $\begin{array}{l}\text { Country of } \\
\text { publisher }\end{array}$ & URL address \\
\hline Asia-Pacific Journal of Social Quality & Korean Association of Converging Humanities & Korea & http://www.converging.eu/ForContributors/01.php \\
\hline International Journal of Martial Arts & Kyung Hee University, Korea & Korea & http://www.injoma.com/sub05.htm \\
\hline Journal of Korean Literature and Culture & Harvard University & US & $\begin{array}{l}\text { https://www.abebooks.com/9780988692800/Azales- } \\
\text { Journal-Korean-Literature-Culture-0988692805/plp }\end{array}$ \\
\hline Journal of Korean Studies & University of California, Los Angeles & US & $\begin{array}{l}\text { http://www.international.ucla.edu/korea/publications/ } \\
\text { journal }\end{array}$ \\
\hline The Journal of Korean Studies & Columbia University & US & http://jks.weai.columbia.edu/submission-guidelines \\
\hline $\begin{array}{l}\text { Korean Journal for Han Character and } \\
\text { Classical Written Language Education }\end{array}$ & $\begin{array}{l}\text { Korean Association for Han Character and } \\
\text { Classical Written Language Education }\end{array}$ & Korea & http://www.studyhanja.net/html/sub3_03.html \\
\hline Pacific Affairs & British Columbia University & Canada & $\begin{array}{l}\text { http://www.pacificaffairs.ubc.ca/submissions/roman- } \\
\text { ization-guide/ }\end{array}$ \\
\hline
\end{tabular}

\section{Reference Styles}

This section presents an explanation of how to romanize Korean according to various reference styles.

\section{Council of Science Editors style}

In the eighth edition of Scientific style and format [4], International Organization for Standardization (ISO) standards were introduced for the romanization of Korean in a document entitled "Information and documentation: transliteration of Korean script into Latin characters. ISO/TR 11941:1996." However, this document has been withdrawn. It is difficult to determine why it was withdrawn. I can think of 2 possible reasons. First, no agreement exists between the South and North Korean governments regarding this standard. Second, the Korean government proposed another system in 2000, which is very distinct from the previously well-known McCune-Reischauer system. Council of Science Editors (CSE) also introduced the American Library Association and Library of Congress (ALA-LC) Romanization Tables, first drafted in 1997, which remains widely used with updates. This system continues to follow the McCune-Reischauer system of romanizing Korean, with certain exceptions. CSE does not permit journal title abbreviations of romanized words from Korean. No example of romanized Korean is presented, either in the text or in the end-references.

Usage in end-references depends on which system of reference is adopted: citation-sequence, name-year, or citationname. The citation-name system is almost the same as the ci- tation-sequence system, but the cited articles are arrayed in the end-references according to the author's name, not the order of appearance in the main text. The citation-sequence system is used in American Medical Association (AMA) style, the National Library of Medicine (NLM) style guide, and Institute of Electrical and Electronics Engineers (IEEE) style, while the name-year system is used in American Psychological Association (APA) style.

\section{American Chemical Society style}

This guide does not provide any information on the romanization of Korean. There is a mention of Korean surnames. In American Chemical Society (ACS) style, the citation-sequencing or name-year system can be used according to the journal's policy. Most journals adopt the citation-sequence system, and journal articles can be listed as below after romanization according to the McCune-Reischauer system:

Author's example

맹광호. 한국의 공중보건 1 세기. 의사학 $1999,8,127-36$.

Maeng, K.H. Hangugŭi kongjungbogŏn 1 segi [One hundred years' history of public health activities in Korea]. Korean J Med Hist 1999, 8, 127-36.

\section{AMA style}

In this style guide, there is no mention of the romanization of Korean characters. There is only one statement that if any words are romanized from a non-Roman alphabet, they should be italicized. Moreover, there is no example of roman- 
ized Korean, either in the text or in end-references [5]. Below is the author's example of an end-reference:

Maeng KH. Hangugŭi kongjungbogŏn 1 segi [One hundred years' history of public health activities in Korea]. Korean J Med Hist 1999;8:127-36.

\section{NLM style guide}

This guide also follows the ALA-LC Romanization Table for Korean. Of the various reference guides, it provides the most precise example of the romanization of Korean, as follows [6]:

For a journal title in a non-roman alphabet: romanize (write in the roman alphabet) titles in Cyrillic, Greek, Arabic, Hebrew, or Korean or in a character-based language (Chinese, Japanese). A good authority for romanization is the ALALC Romanization Tables [7] for example.

Taehan Kan Hakhoe Chi. Seoul (Korea): Taehan Kan Hakhoe. Vol. 1, 1995 - Vol. 9, No. 4, Dec 2003. Korean.

It is not NLM practice, but you may translate journal titles in character-based languages for example, Korean Journal of Hepatology. Seoul (Korea): Taehan Kan Hakhoe. Vol. 1, 1995 - Vol. 9, No. 4, Dec 2003. Korean

Provide an English translation after the original language or romanized title when possible; place translations in square brackets.

Author's example of a book from “이남희, 『조선후기 잡과 중인 연구』, 이회, 2000.”

Lee NH. Chosŏn hugi chapkkwa-chung-in yŏngu [A study of Chapkwa-Chungin in the late chosŏn Period]. Ihoei, 2000 .

It is not mandatory to add a translated title after the romanized title; however, it is better if possible to facilitate a clear understanding of the meaning of the romanized Korean text. If a journal article is cited, the author's example is given:

Maeng KH. Hangugŭi kongjungbogŏn 1 segi [One hundred years' history of public health activities in Korea]. Ǔisahak. 1999;8(2):127-36.

In the NLM style guide, if the official title of the journal is not in English, the original journal title is romanized; therefore, Uisahak is used instead of the Korean Journal of Medical History.

\section{APA style}

APA style also uses the McCune-Reischauer system for the romanization of Korean [8,9]. Therefore, it is recommended to refer to ALA-LC Romanization Tables [7]. APA style de- scribes the romanization of Korean more specifically than other styles.

\section{Translation of title}

Ch'ae Paek. (2008). Kŭndae Minjokchuŭi ŭi hyŏngsŏng kwa kaehwagi ch'ulp'an [Emergence of nationalism and publishing during the Enlightenment period in modern Korea]. Han'gukŏllon chŏngbo hakpo, 41, 7-40.

Aauthor's example

Maeng Kwang Ho. (1999). Hangugŭi kongjungbogŏn 1 segi [One hundred years' history of public health activities in Korea]. Uisahak, 8, 127-36.

Inclusion of Korean script or Chinese characters Han Paek-kyŏm 韓百謙. (1982). Tongguk chiri chi東國地 理志 [Geographic records of the Eastern Kingdom]. Seoul: Iljogak.

\section{IEEE style}

This system contains no specific guidance for the romanization of Korean [10]. It stated that for guidance in grammar and usage not included in that manual, the Chicago manual of style, published by the University of Chicago Press, should be consulted. Therefore, the following example based on the Chicago manual of style can be used [11].

Book example

Kang Ubang 姜友邦, Wonyung kwa chohwa:Han'guk kodae chogaksa ŭi wŏlli 圓融과調和: 韓國古代彫刻史의 原理 [Synthesis and harmony: Principle of the history of ancient Korean sculpture] (Seoul: Yŏrhwadang, 1990), 190197.

Journal example

Han, T’aesik 韓泰植. “Kyŏnghŭng ŭi saengae e kwanhan chae kochal" 憬興의 生涯에 관한 재고찰[Re-examination of the life of Kyŏnghŭng]. Pulgyo hakpo 佛敎學報 28 (1991): 187-213.

\section{How to Romanize Korean and How to Cite in the End-references}

First, the editor should declare which system he or she will choose: the 2000 Korean government's system or the 1939 McCune-Reischauer system. This decision is up to the editor. However, if a journal is related to geography or military studies, the McCune-Reischauer system is recommended. The Korean Army cannot help but use the McCune-Reischauer system because the United States Army uses this system. If 


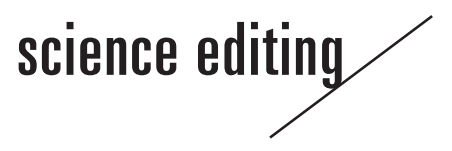

there are differences in the terminology used for geographic features, cultural properties, man-made structures, and administrative units between 2 countries' armies, it will become difficult to execute military operations together. They should use the same map with the same names of geographic and other features. Second, the reference style should be the journal's house style. Therefore, the editor should clarify the style in the instructions to authors. If it is clearly stated, authors and manuscript editors should use the selected style.

\section{Romanization of Chinese Characters in Korean}

Chinese characters have been used in written Korean-language sources from the early period of Korean history, for more than 2000 years. To this day, it is common to find Chinese characters in Korean documents. Chinese characters are romanized according to the Korean pronunciation. For example, “김부식金富軾. 삼국사기三國史記, 1145 ” can be romanized as follows according to NLM style: Kim BS. Samguksagi [History of three kingdoms]. [publisher unknown]. 1145.

If a document is in Chinese characters and was published in China or Japan, romanization usually follows the Pinyin romanization standard if published in China [7], and the Kunrei-shiki romanization if published in Japan. Because this is another topic, it should be dealt with in another piece [12].

\section{How to Treat the 2 Systems of Romanization of Korean}

Both for novice editors and experienced editors, romanization is not simple. Editors should understand the 2 systems used at present in Korea. If manuscript editors can help other editors, this issue can be solved without difficulty. Why did the Korean government not propose the ISO standard for the romanization of Korean? This is a very difficult topic, because when the Korean government revised the system in 2000, essential members participating in the revision process did not hear voices from foreign countries. Many scholars on Korean studies throughout the world were disappointed at that time. I, as an editor, also opposed and presented an opposing opinion at the public hearing; however, they did not listen to me. I strongly suggest that special symbols can be omitted in everyday life. Another problem is the issue of agreement with the North Korean government, because Korean is also the official language of North Korea. Unfortunately, I suppose that discrepancies of this sort will not be resolved in the foreseeable future. Outside of Korea, the McCune-Reischauer system still prevails among international journals. Editors should select a single style. Oh [13] stated that for cataloging in libraries in Korea, adopting the Korean government's system of roman- ization may cause confusion in information retrieval systems. However, 10 years later, in libraries in Korea, in cataloging, most librarians follow the Korean government's system.

\section{Conclusion}

To facilitate a more accurate understanding of the romanization of Korean, editors and manuscript editors should understand the 2 systems used in Korea: the Korean government's romanization system and the McCune-Reischauer system. An editor should select one of these systems and use it consistently. In terms of presenting romanized text, the journal's house style should be followed, based on international reference citation styles such as the CSE, ACS, AMA, APA, and IEEE styles and the NLM style guide. Chinese characters in documents published in Korea should be romanized according to the Korean pronunciation. For a more detailed understanding, the list of references provided herein will be useful.

\section{Conflict of Interest}

No potential conflict of interest relevant to this article was reported.

\section{References}

1. National Institute of Korean Language, Republic of Korea. Romanization of Korean [Internet]. Seoul: National Institute of Korean Language, Republic of Korea; 2000 [cited 2017 July 24]. Available from: http://www.korean.go.kr/

2. McCune GM, Reischauer EO. Romanization of Korean language based upon it phonetic structure. Trans Korea Branch R Asiatic Soc 1939;29:1-55.

3. Park IS. History of the national licensing examination for the health professions under the Japanese GovernmentGeneral of Korea (1910-1945). J Educ Eval Health Prof 2015;12:21. https://doi.org/10.3352/jeehp.2015.12.21

4. Council of Science Editors. Scientific style and format: the CSE manual for authors, editors, and publishers. 8th ed. Chicago, IL: Council of Science Editors; 2014.

5. American Medical Association. AMA manual of style: a guide for authors and editors. 10th ed. Oxford, NY: Oxford University Press; 2007.

6. Patrias K, Wendling D; National Library of Medicine. Citing medicine: the NLM style guide for authors, editors, and publishers. 2nd edition [Internet]. Bethesda, MD: National Library of Medicine; 2007. Available from: https:// www.ncbi.nlm.nih.gov/books/NBK7256/

7. Library of Congress. ALA-LC Romanization Tables [Internet]. Washington, DC: Library of Congress; 2009 [cited 
2017 Jul 26]. Available from: https://www.loc.gov/catdir/ cpso/roman.html

8. University of British Columbia. American Psychological Association style guide for Korean source [Internet]. Vancouver, BC: University of British Columbia; 2016 [cited 2017 Jul 26]. Available from: http://wiki.ubc.ca/images/1/16/APA_Guide_Korean.pdf

9. Yale University Library. Quick guide on citation style for Chinese, Japanese and Korean sources: APA example [Internet]. New Haven, CT: Yale University Library; 2017 [cited 2017 Jul 26]. Available from: http://guides.library. yale.edu/c.php?g=296262\&p=1974231

10. IEEE. IEEE editorial style manual [Internet]. Piscataway, NJ: IEEE; 2016 [cited 2017 Jul 26]. Available from: https:// www.ieee.org/documents/style_manual.pdf

11. Yale University Library. Quick guide on citation style for Chinese, Japanese and Korean sources: Chicago Manual examples [Internet]. New Haven, CT: Yale University Library; 2017 [cited 2017 Jul 26]. Available from: http:// guides.library.yale.edu/c.php?g $=296262 \& p=1974227$

12. International Organization for Standardization. ISO 3602:1989. Documentation: romanization of Japanese (kana script) [Internet]. Geneva: International Organization for Standardization; 1989 [cited 2017 Jul 26]. Available from: https://www.iso.org/standard/9029.html

13. Oh KM. A study on romanization rules and practices of the international institutions for Korean language materials. J Korean Soc Inf Manag 2007;24:33-51. 